Article

\title{
High Frequency of ERBB2 Activating Mutations in Invasive Lobular Breast Carcinoma with Pleomorphic Features
}

\author{
Juan Manuel Rosa-Rosa ${ }^{1, *}$, Tamara Caniego-Casas ${ }^{2}$, Susanna Leskela ${ }^{1,2}$, Eva Cristobal ${ }^{2}$, \\ Silvia González-Martínez ${ }^{3}$, Esther Moreno-Moreno ${ }^{4}$, Elena López-Miranda ${ }^{3}$, Esther Holgado ${ }^{3}$, \\ Belén Pérez-Mies ${ }^{2,4,5}$, Pilar Garrido ${ }^{1,2,3,5}$ and José Palacios $1,2,4,5, *$ (D) \\ 1 CIBER-ONC, Instituto de Salud Carlos III, 28029 Madrid, Spain; susanna.leskela@gmail.com (S.L.); \\ pilargarridol@gmail.com (P.G.) \\ 2 Instituto Ramón y Cajal de Investigación Sanitaria, 28034 Madrid, Spain; \\ tamara880723@hotmail.com (T.C.-C.); evamaria.cristobal@salud.madrid.org (E.C.); \\ bperezmies@gmail.com (B.P.-M.) \\ 3 Department of Medical Oncology, Hospital Ramón y Cajal, 28034 Madrid, Spain; \\ silviagonzalezmartinezbio@gmail.com (S.G.-M.); elemiranda@hotmail.com (E.L.-M.); \\ eholgadomartin@gmail.com (E.H.) \\ 4 Department of Pathology, Hospital Ramón y Cajal, 28034 Madrid, Spain; emorenomoreno92@gmail.com \\ 5 Facultad de Medicina, Universidad de Alcalá de Henares, 28029 Madrid, Spain \\ * Correspondence: juanmanuel.rosa@salud.madrid.org (J.M.R.-R.); jose.palacios@salud.madrid.org (J.P.)
}

Received: 16 November 2018; Accepted: 7 January 2019; Published: 11 January 2019

\begin{abstract}
Background: Characterisation of molecular alterations of pleomorphic lobular carcinoma (PLC), an aggressive subtype of invasive lobular carcinoma (ILC), have not been yet completely accomplished. Methods: To investigate the molecular alterations of invasive lobular carcinoma with pleomorphic features, a total of 39 tumour samples (in situ and invasive lesions and lymph node metastases) from 27 patients with nuclear grade 3 invasive lobular carcinomas were subjected to morphological, immunohistochemical and massive parallel sequencing analyses. Results: Our observations indicated that invasive lobular carcinomas with pleomorphic features were morphologically and molecularly heterogeneous. All cases showed absence or aberrant expression of E-cadherin and abnormal expression of $\beta$-catenin and p120. CDH1 (89\%), PIK3CA (33\%) and ERRB2 (26\%) were the most common mutated genes. $E R B B 2$ mutations preferentially affected the tyrosine-kinase activity domain, being the most frequent the targetable mutation p.L755S (57\%). We also observed higher frequency of mutations in ARID1B, KMT2C, MAP3K1, TP53 and ARID1A in PLC than previously reported in classic ILC. Alterations related to progression from in situ to invasive carcinoma and/or to lymph node metastases included TP53 mutation, amplification of PIK3CA and CCND1 and loss of ARID1A expression. Conclusions: The high frequency of $E R B B 2$ mutations observed suggests that $E R B B 2$ mutation testing should be considered in all invasive lobular carcinomas with nuclear grade 3 .
\end{abstract}

Keywords: lobular carcinoma; pleomorphic; NGS; ERBB2

\section{Introduction}

Invasive Lobular Carcinoma (ILC) is the second most prevalent histologic subtype of breast cancer, constituting up to 10-15\% of all cases [1]. Interestingly, the incidence of ILC has increased significantly compared with that of invasive ductal carcinoma of non-special type (IDC) since the 1980s, probably due to the use of hormone replacement therapy during menopause [2]. There are several variants of ILC with lack of cell-cell cohesion as a common feature: classic ILC, solid ILC, alveolar ILC, tubulo-lobular 
ILC and pleomorphic ILC (PLC) [2]. PLC is an uncommon but clinically important form of ILC, it was first described as a more aggressive variant of ILC compared with the classic subtype [3,4]. In terms of epidemiology, PLC represents less than $1 \%$ of all breast cancer and about $15 \%$ of ILC and it is associated with older age and menopausal status. Despite having a growth pattern similar to classic ILC, PLC shows both a higher degree of cellular atypia and pleomorphism and a higher mitotic rate, than classic ILC. Additionally, PLC may show apocrine or histiocytoid differentiation and it may be composed of signet ring cells [2].

The key molecular hallmark of ILC is the loss of the epithelial specific cell-cell adhesion molecule E-cadherin, encoded by $C D H 1$ [5]. Genetic mutations involving loss of function have been described in all subtypes of ILC [6], ranging between $50-60 \%$ of ILC cases. Deleterious mutations in CDH1 are usually accompanied by the loss of 16q, where the gene is located [7]. Thus, there is a complete loss of E-cadherin, conferring the highly non-cohesive morphological characteristics of this type of tumour [1]. In addition, the loss of E-cadherin expression is also observed in LCIS, so CDH1 mutations/alterations are expected to be early events in this subtype.

More recently, three large studies based on next generation sequencing (NGS) techniques have reported the molecular characteristics of ILC. These studies have demonstrated that there are molecular differences between ILC and IDC and that the PI3K pathway is the most frequently altered due to mutations in PIK3CA, PTEN or AKT1 [8]. Although not specifically stated, most cases included in this series corresponded to classic ILC. Out of these three studies, only Desmedt et al. [9] tried to associate molecular alterations with specific morphological features, such as nuclear pleomorphism, growth pattern or mitotic index.

At present we partially lack molecular information regarding ILC histological subtypes. PLC has been characterised previously using immunohistochemistry, single gene analysis and microarray-based comparative genomic hybridization (aCGH) [9-12]. These analyses have confirmed the loss of E-cadherin expression and 16q loss of heterocigosity (LOH) [9] in PLC. Additionally, PLC carries molecular alterations typical of high-grade IDC, such as HER2 and TP53 alterations, more frequently than classic ILC [10-12]. Lastly, two NGS studies have specifically analysed the molecular alterations in PLC in two small series of 8 and 16 tumours respectively [13,14].

PLC is more aggressive than other forms of ILC. In spite of previous studies, molecular characterisation of PLC has not yet been fully accomplished. Thus, we have selected PLC tumours from 27 patients to be characterised by NGS using a specific gene panel to target the most frequently mutated genes and altered chromosomal regions in breast cancer. To gain insight into the molecular alterations involved in tumour progression, we analysed the molecular differences between the in-situ and the invasive components in 7 tumours and between the invasive component and the lymph node metastasis in 4 tumours.

\section{Materials and Methods}

Compliance with Ethical Standards: All procedures performed in studies involving human participants were in accordance with the ethical standards of the institutional and national research committee and with the 1964 Helsinki declaration and its later amendments or comparable ethical standards.

\subsection{Case Selection}

A total of 39 tumour samples from 27 patients diagnosed between 2010 and 2017 were selected from the Pathology Department in Ramón y Cajal Hospital (Madrid, Spain). Cases were included in the study if they had a growth pattern suggestive of ILC, nuclear grade 3, absence or abnormal E-cadherin expression (see Section 3) and tissue available for additional immunohistochemical and molecular studies (Supplementary Table S1). The study included 27 invasive, 7 in-situ, 4 lymph node metastasis and 1 relapse lesions. 
Histological evaluation was performed according to WHO recommendations and included tumour size, growth pattern (classic, solid, alveolar or trabecular) and cellular morphology (classic, apocrine or histiocytoid). All cases were graded according to the three-tiered Nottingham histological grading system [2].

\subsection{Immunohistochemistry}

The antibodies used are shown in Supplementary Table S2. Immunostaining was performed using the EnVision detection system (K5007, Dako, Glostrup, Denmark). A cut-off value of 1\% was used to define ER, PR or AR positivity. Ki67 index was defined as the percentage of positive cells independently of intensity. HER2 expression was interpreted according to 2018 American Society of Clinical Oncology and the College of American Pathologists (ASCO-CAP) guidelines [15]. E-cadherin, $\beta$-catenin and p120 expression was classified as membranous, cytoplasmic or nuclear. Membranous expression was considered to be preserved (intense expression in more than $75 \%$ of neoplastic cells), reduced or absent as previously reported [16]. For cyclin D1, ARID1A and ARID1B nuclear proteins, a $5 \%$ cut-off value was used to define positivity. The total number of CD8+ T lymphocytes were visually measured in each tumour microarray, counting intratumoural and stromal lymphocytes altogether. Tumours were considered to be PDL1-positive if PDL1 expression was observed in more than $1 \%$ of neoplastic cells.

Tumours were classified in different intrinsic subtypes as Luminal (see Section 3), HER2-enriched (HER2-positive and ER- and PgR-negative) and Triple Negative (HER2-, ER- and PgR-negative) according to Curigliano et al. criteria [17]. Luminal tumours were classified according to Maisonneuve et al. [18] criteria as follow: (1) Luminal A-like tumours are ER-positive and HER2-negative with low Ki-67 expression $(<14 \%)$ or with intermediate Ki-67 expression (14\% to $19 \%)$ and high PgR levels $(\geq 20 \%)$; (2) Luminal B-like (HER2-negative) tumours are ER-positive and HER2-negative with intermediate Ki-67 expression (14\% to $19 \%$ ) and low PgR levels ( $<20 \%$ ) or with high Ki-67 expression $(\geq 20 \%)$.

\subsection{Fluorescent In-Situ Hybridisation (FISH)}

HER2 equivocal cases (2+) were subjected to fluorescent in situ hybridisation, using the path vision HER-2 DNA Probe Kit (Abbot Laboratories, City, Country). Results were interpreted according to 2018 ASCO-CAP guidelines.

\subsection{Massive Parallel Sequencing}

To obtain DNA mainly from tumour cells, samples from different lesions were obtained by "punching" paraffin blocks in selected areas previously marked on haematoxylin/eosin (H\&E) slides. QIAamp DNA FFPE Tissue Kit (Qiagen, Valencia, CA, USA) was used to extract DNA from all samples. Quality of DNA samples was measured using TapeStation (Agilent 2200 TapeStation, Santa Clara, CA, USA), whereas quantification was done by QUBIT 2.0. (Thermo Fisher Scientific Qubit 2.0 Fluorometer, Waltham, MA, USA).

A custom gene panel was designed using SureDesign platform by Agilent Tech. (Santa Clara, CA, USA) to consistently target 34 genes (AKT1, ARID1A, ARID1B, BRCA1, BRCA2, CASP8, CCND1, CDH1, ERBB2, ESR1, FGFR1, GATA3, GRB7, GSDMB, MAP2K4, KRAS, MAP3K1, MLL3, MYC, NCOR1, NF1, PGAP, PIK3CA3, PNMT, PTEN, RB1, SF3B1, STARD3, TBX3, TCAP, TP53, VGLL1, ZNF217, ZNF703) and regions in chromosome 8 (targeting amplification of FGFR1 and MYC), chromosome 11 (targeting amplification of CCND1), chromosome 17 (targeting amplification of ERBB2) and chromosome 20 (targeting amplification of ZNF217). For library construction, a modified protocol for Agilent SureSelect ${ }^{\mathrm{QXT}}$ was selected [19], based on the enzymatic fragmentation of DNA and subsequent probe-mediated hybridisation capture [20]. Sequencing of equimolar libraries was performed using the Miseq v2 $2 \times 150$ bp method by Illumina Inc., San Diego, CA, USA.

Bioinformatics analysis was carried out using a specific pipeline using Novoalign (http:/ /www. novocraft.com/products/novoalign/) as aligner and VarScan [21] as variant-caller, with no filters. 
Variant annotation was performed using the VEP from Ensembl version 88 (http:/ / www.ensembl.org/ info/docs/tools/vep/index.html), which corresponds to hg38 version of the human reference genome. Variants were latterly filtered using the functional information (taking only deleterious variants), the variant allele frequency $(>0.05)$ and the strand-bias from both the variant and the reference allele. If normal tissue was available, those variants also present in the normal component were ruled out. Finally, visual inspection was performed as the final selection criterion. In addition, dubious variants were confirmed or ruled out using Sanger sequencing. CONTRA package [22] was used to analyse the genome instability using a baseline obtained from several normal diploid FFPE samples (from normal Fallopian tubes) used as control samples.

\subsection{Estimation of LOH Status in CDH1}

Since our gene panel was not designed to identify losses on the $C D H 1$ chromosomal region, we aimed to estimate the $\mathrm{LOH}$ status of $\mathrm{CDH1}$ for each sample. Although two different polymorphisms (rs3743674 and rs1801552) were constitutively identified in our tumour set, they failed to estimate the $\mathrm{LOH}$ status in the region, due to the allele frequencies in the population and the apparently linked status of their alleles.

Therefore, we developed a simple formula based on the percentage of tumour cells:

$$
\mathrm{Ti}=\frac{\text { \%tumour }_{\text {cells }}}{2-\% \text { tumour }_{\text {cells }}}
$$

This $T i$ (Tumoural index) was then compared to the frequency of the mutated allele in our NGS data, giving an approximation of the $\mathrm{LOH}$ status of $\mathrm{CDH1}$. Considering that the percentage of tumour cells could be overestimated and that the frequency of the variant allele observed by NGS data could be underestimated, we considered a $-0.17 \times$ Ti deviation as acceptable. See Supplementary File 1 for complete mathematical explanations.

\section{Results}

\subsection{Clinicopathological Features}

Clinicopathological and immunohistochemical features of all samples are shown in Supplementary Table S1 and summarised in Table 1. The average age at diagnosis was 63 y/o (range 39 to 87), being $63 \%$ of patients older than 60 years.

All 27 PLCs had high nuclear grade (G3) and no tubular formation, hence all were classified as histologic grades $2(63 \%)$ or $3(37 \%)$, depending on their mitotic count. The predominant pattern of growth was trabecular (48\%), followed by solid (37\%) and classic (15\%). Regarding cytomorphology (see Figure 1), 7 cases (26\%) showed histiocytoid differentiation and 3 cases $(11 \%)$ apocrine differentiation. This series included an uncommon case rich in osteoclast-like cells [23]. According to classification criteria $[17,18], 17$ PLCs ( 63\%) were luminal A, 5 ( 19\%) PLCs were luminal B, 4 ( 15\%) PLCs were triple negative and $1(\sim 4 \%)$ PLC was HER2-enriched.

As to E-cadherin expression, 23 PLCs showed complete absence of expression. The remaining four cases showed different patterns of abnormal expression (see Figure 2): three cases had aberrant cytoplasmic expression and one PLC showed severe reduction of membranous expression.

Consistent with the abnormal E-cadherin expression patterns described, all cases showed abnormal $\beta$-catenin and p120 expression patterns. Regarding $\beta$-catenin, 63\% of PLCs showed complete absence of expression and the remaining cases had abnormal cytoplasmic expression. p120 cytoplasmic expression was observed in $92 \%$ of the cases and of note, $22 \%$ of PLCs displayed both cytoplasmic accumulation and nuclear expression. 
Table 1. Clinicopathological features of the 27 patients.

\begin{tabular}{cccc}
\hline Age at Diagnosis (Years) & \#Cases & Receptor Status & \#Cases \\
\hline$<35$ & 0 & ER+ & 20 \\
$35-49$ & 4 & ER- & 7 \\
$50-69$ & 13 & PgR+ & \\
$70+$ & 10 & PgR- & 16 \\
Tumour size & & & 11 \\
N/A & 2 & AR+ & 22 \\
$<1$ cm & 1 & AR- & 5 \\
$1-2$ cm & 7 & Ki67 & \\
$2-5$ cm & 15 & $<14$ & 20 \\
$>5$ cm & 2 & $14-30$ & 6 \\
N0 & & $30+$ & 1 \\
N1 & 11 & HER2 & 26 \\
N3 & 11 & $0 /+/++$ & 1 \\
NX & 4 & +++ & \\
Tumour grade & 1 & E-cadherin status & 23 \\
G2 & 17 & - & 4 \\
G3 & 10 & aberrant & \\
Subtype & & & \\
Luminal A & 17 & & \\
Luminal B & 5 & & \\
Triple Negative & 4 & & \\
HER2 enriched & 1 & & \\
\hline
\end{tabular}

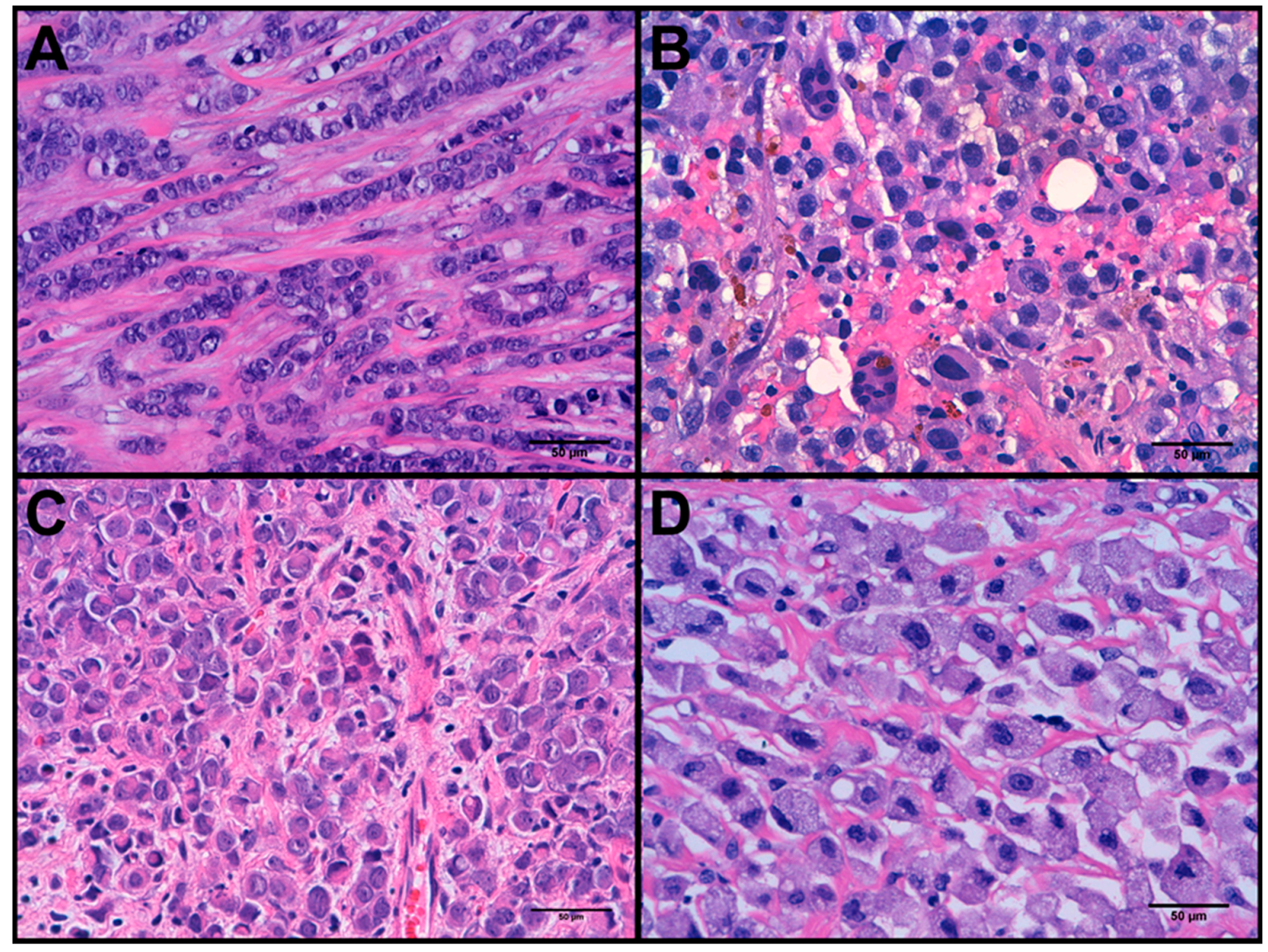

Figure 1. Histological variants of pleomorphic lobular carcinomas (PLC). (A) PLC with no other cell type. (B) PLC with osteoclast-like giant cells. (C) Apocrine PLC. (D) Histiocytoid PLC. 


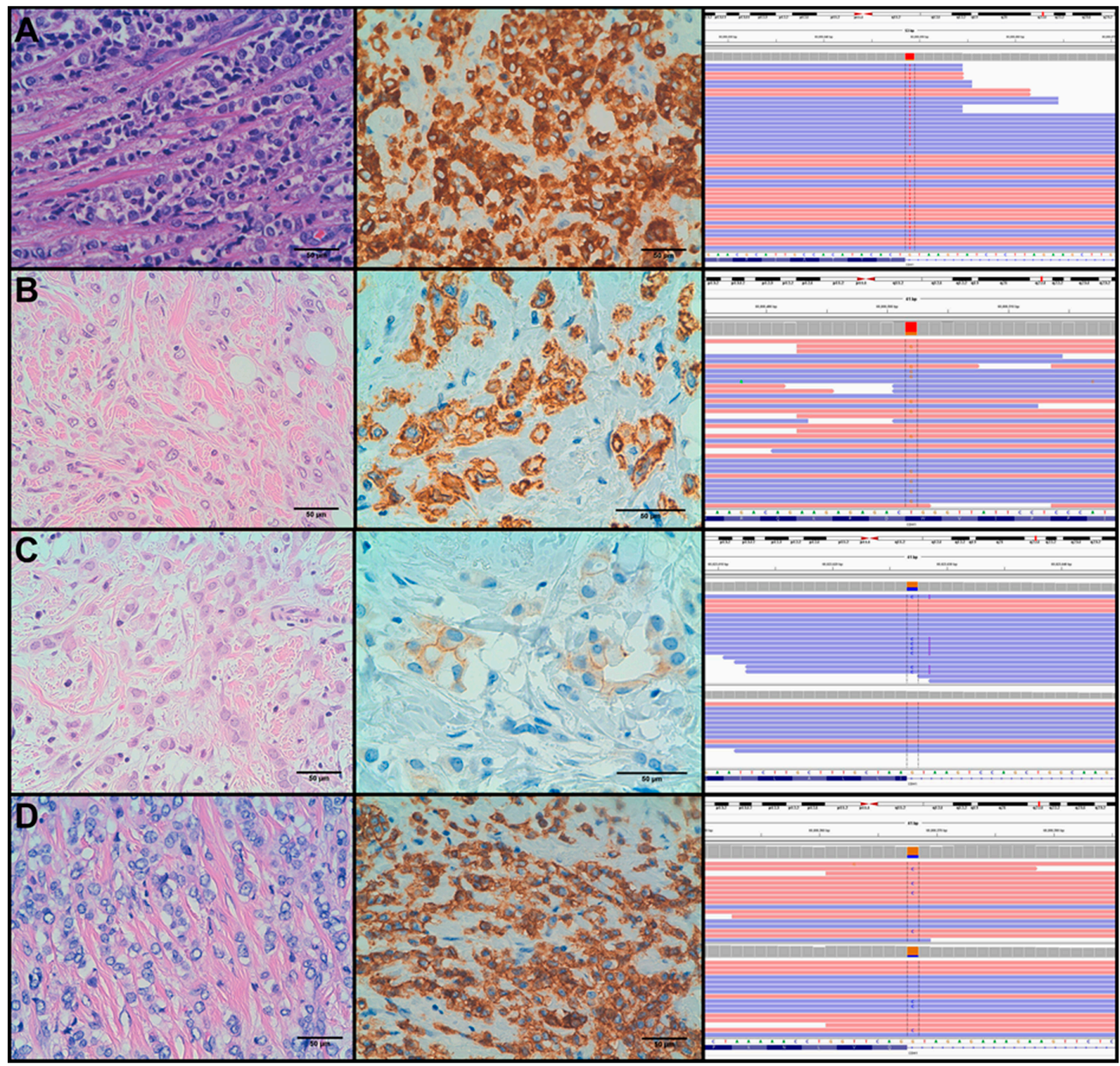

Figure 2. PLC cases showing aberrant expression of E-cadherin: PLC-5 (A), PLC-7 (B), PLC-18 (C) and PLC-22 (D). From left to right: H\&E, E-cadherin staining and IGV view of CDH1 mutations. In PLC-18 and PLC-22, reads and bases are shown for invasive (top) and in-situ (bottom) components.

Regarding other markers, no cases showed p53 overexpression or a null pattern of expression. All tumours expressed ARID1B and CK19. 70\% of PLCs expressed androgen receptor and 26 out of 27 (92\%) PLCs expressed ARID1A and cyclin D1. PDL1 was positive in only six PLCs, considering $1 \%$ as the cut-off. The average number of CD8+ lymphocytes per tumour was 35 (range 7-90).

\subsection{Mutation Analysis}

Variant analysis results are shown in Supplementary Table S3. A total of 78 somatic variants and 8 amplifications were identified among the 27 invasive samples. As expected, most samples ( 24 out of $27,88.89 \%$ ) had mutations in CDH1 (see Table 2), the most frequently mutated gene in this series. The distribution of mutations across $C D H 1$ is shown in Figure 3A. Interestingly, 12 out of the $25(48 \%)$ mutations were indels, $6(24 \%)$ mutations were located in the conserved donor site of splice regions, $4(16 \%)$ were nonsense mutations and $2(8 \%)$ were missense mutations. According to the wTi we calculated for 24 cases in which the percentage of tumour cells could be estimated (see Supplementary Table S4), we considered that up to 18 out of 21 (85\%) cases with mutations in CDH1 could probably also carry $\mathrm{LOH}$ in the region. 
Table 2. Comparative results with published data for gene mutation frequency.

\begin{tabular}{|c|c|c|c|c|c|c|}
\hline \multirow[b]{2}{*}{ Gene } & \multirow{2}{*}{$\begin{array}{l}\text { Number of Mutations } \\
\text { Found in Current Study }\end{array}$} & \multicolumn{5}{|c|}{$\%$ Cases Mutated } \\
\hline & & $\begin{array}{l}\text { Current Study } \\
(\text { PLC, } n=27)\end{array}$ & $\begin{array}{c}\text { Zhu et al. [14] } \\
(\text { PLC, } n=17)\end{array}$ &  & $\begin{array}{l}\text { Michaut et al. [24] } \\
\quad(\text { ILC, } n=144)\end{array}$ & $\begin{array}{l}\text { Ciriello et al. [1] } \\
\quad(\text { ILC, } n=127)\end{array}$ \\
\hline CDH1 & 25 & $89 \%$ & $59 \%$ & $65 \%$ & $43 \%$ & $65 \%$ \\
\hline PIK3CA & 12 & $33 \%$ & $53 \%$ & $43 \%$ & $35 \%$ & $48 \%$ \\
\hline ERBB2 & 7 & $26 \%$ & $18 \%$ & $5 \%$ & $4 \%$ & $4 \%$ \\
\hline ARID1B & 6 & $22 \%$ & - & $0 \%$ & $5 \%$ & $6 \%$ \\
\hline KMT2C & 5 & $19 \%$ & $35 \%$ & $8 \%$ & $10 \%$ & $7 \%$ \\
\hline MAP3K1 & 5 & $19 \%$ & $35 \%$ & $5 \%$ & $5 \%$ & $6 \%$ \\
\hline TP53 & 5 & $19 \%$ & $12 \%$ & $7 \%$ & $4 \%$ & $8 \%$ \\
\hline ARID1A & 4 & $15 \%$ & $6 \%$ & $6 \%$ & $7 \%$ & $17 \%$ \\
\hline CCND1_amp & 3 & $11 \%$ & $12 \%$ & $38 \%$ & $15 \%$ & $17 \%$ \\
\hline AKT1 & 2 & $7 \%$ & $6 \%$ & $4 \%$ & $5 \%$ & $2 \%$ \\
\hline FGFR1_amp & 2 & $7 \%$ & - & $25 \%$ & $8 \%$ & $9 \%$ \\
\hline GATA3 & 2 & $7 \%$ & $6 \%$ & $7 \%$ & $5 \%$ & $5 \%$ \\
\hline NF1 & 2 & $7 \%$ & $23 \%$ & $0 \%$ & $4 \%$ & $4 \%$ \\
\hline TBX3 & 2 & $7 \%$ & $23 \%$ & $13 \%$ & $8 \%$ & $9 \%$ \\
\hline ERBB2_amp & 1 & $4 \%$ & $6 \%$ & $0 \%$ & $4 \%$ & $7 \%$ \\
\hline PIK3CA_amp & 1 & $4 \%$ & - & $0 \%$ & $1 \%$ & $2 \%$ \\
\hline BRCA2 & 1 & $4 \%$ & $0 \%$ & $2 \%$ & $4 \%$ & - \\
\hline CASP8 & 1 & $4 \%$ & - & $0 \%$ & $1 \%$ & $1 \%$ \\
\hline NCOR1 & 1 & $4 \%$ & $23 \%$ & $0 \%$ & $7 \%$ & $6 \%$ \\
\hline PGAP3 & 1 & $4 \%$ & $12 \%$ & $0 \%$ & $0 \%$ & - \\
\hline RB1 & 1 & $4 \%$ & $0 \%$ & $0 \%$ & $3 \%$ & $6 \%$ \\
\hline
\end{tabular}




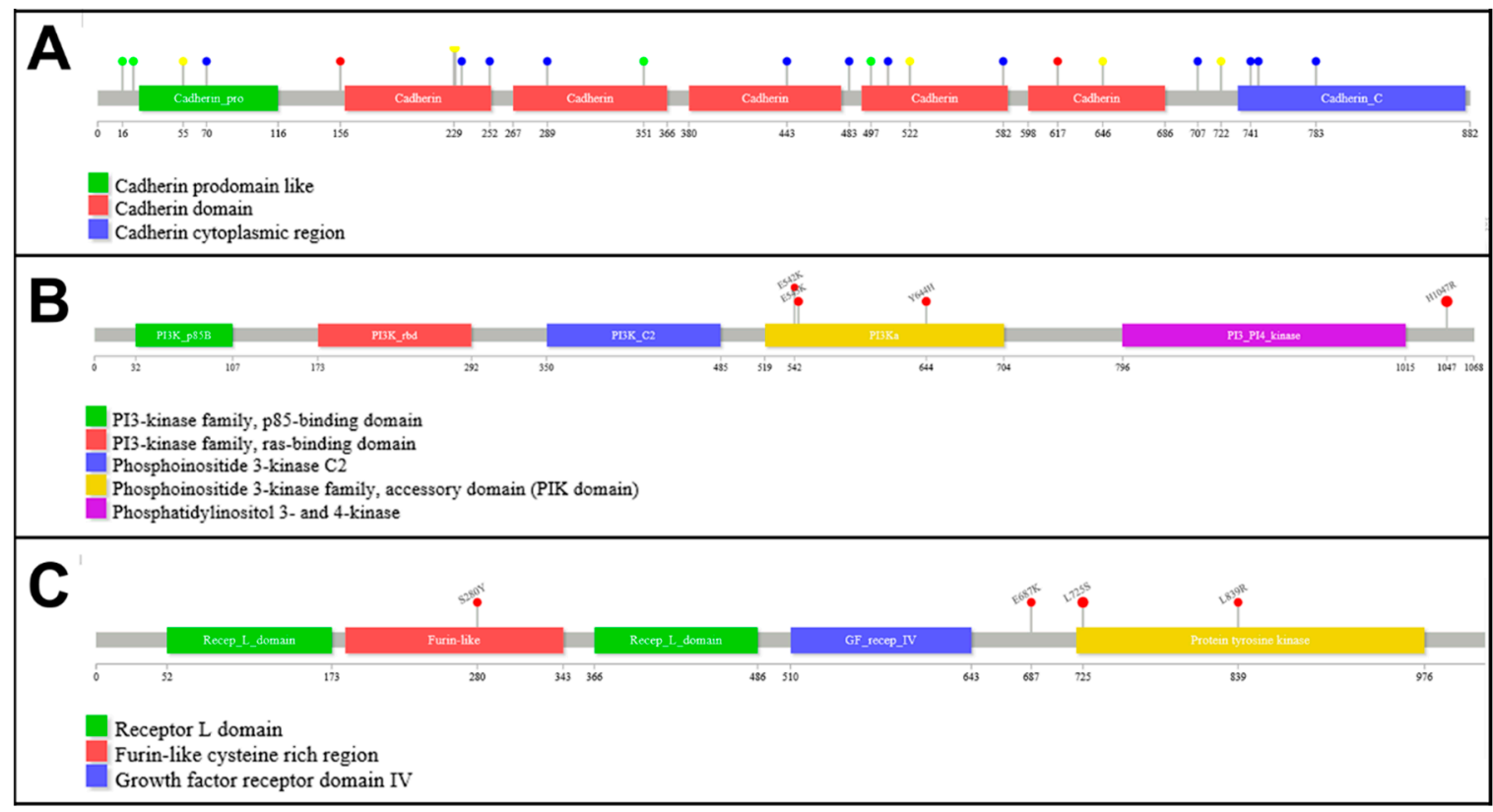

Figure 3. Distribution of somatic mutations along (A) CDH1, (B) PIK3CA and (C) ERRB2 genes. Red lollipops represent missense mutations while green lollipops represent nonsense mutations, blue lollipops represent frameshift mutations and yellow lollipops represent splice-affecting mutations.

Together with CDH1, PIK3CA and ERRB2 were the most common mutated genes in this series and showed mutations in $9(33 \%)$ and $7(26 \%)$ tumours respectively. In addition, both PIK3CA and $E R R B 2$ were amplified in one case respectively. The mutation distribution across PIK3CA and ERBB2 are shown in Figure 3B,C respectively. Most mutations in PIK3CA were found in hotspot positions: p.H1047R (58\%), located in the phosphoinositide-3-kinase domain; and p.E545K (17\%) and p.E542K $(8.5 \%)$, both located in the protein kinase-C homology domain. We also found the same mutation (p.Y644H) in 2 different cases, located in the helical domain, which are probably germline variants. Similarly, we found a predominant hotspot mutation in ERBB2 (p.L755S, 57\%) and other 2 mutations (p.E717K and p.L869R) also affecting the tyrosine-kinase activity domain. We also found a mutation (p.S310Y) located in the binding-site domain.

Mutations in other genes involved in the PI3K/AKT pathway included mutations in MAP3K1 (present in 5 cases, 19\%) and AKT1 (present in 2 cases, 8\%). Mutations in PIK3CA, ERBB2 and AKT1 (present in a total of 16 cases) tend to be mutually exclusive (only cases PLC9 and PLC19 carried mutations in both PIK3CA and ERBB2). In contrast, all MAP3K1 mutations occurred in cases with mutations in PIK3CA or ERBB2.

Mutations in TP53 occurred in 5 cases (18.52\%). Interestingly, whereas 2 cases had mutations producing truncated proteins (c.550_551delGA and p.R342*), the remaining 3 cases displayed missense mutations (p.R175H, p.Y220C and p.E286K) affecting the DNA binding domain.

Mutations in the chromatin remodelling genes ARID1B and ARID1A were found in $6(22 \%)$ and $4(15 \%)$ tumours, respectively. Mutations in both genes were mutually exclusive except in one case (PLC15). Mutations in the gene encoding the histone H3 lysine 4 methyltransferase KMT2C were found in 5 cases $(19 \%)$.

As for amplifications, in addition to the previously mentioned cases with ERBB2 and PIK3CA amplification, we observed that the CCND1 region was amplified in 3 cases (11\%) and FGFR1 in 2 cases $(7 \%)$.

In addition to somatic mutations, we observed a set of infrequent germline variants (Supplementary Table S5) that were overrepresented in our series. The extreme overrepresentation of BRCA2 variants and ERRB2 variant rs141116145 (p.A356D) is noteworthy, since two different cases present the variant allele while the expected number of carriers in our set was 0.05 according 
to Hardy-Weinberg equilibrium (see Supplementary Table S5). Interestingly, PLC13 carried the pathogenic mutation c.3860delA in BRCA2 (confirmed as a germline mutation) and had a family record in which her mother had an ovary carcinoma and her daughter debuted with breast carcinoma at the age of 30 .

\subsection{Molecular Alterations and Histopathological/Immunohistochemical Features}

Regarding the relationship between molecular alterations and IHC features, it is interesting to note that aberrant expression patterns of E-cadherin can be found in CDH1-mutated tumours that can be mistaken with positive E-cadherin staining (see Figure 2). We observed an aberrant expression pattern of E-cadherin in 4 cases: PLC5, PLC7, PLC18 and PLC22 (see Supplementary Table S1 and Figure 2). In 3 out of the 4 cases we identified mutations in $C D H 1$ that affected canonical splicing sites (c.531+1G > C, c.687+1G $>\mathrm{T}$ and c.2164+1G > C), according to in-silico assays (data not shown). The remaining case portrayed a missense mutation (p.W156G) which functional effect on the protein has not been elucidated. On the other hand, we found other three cases (PLC10, PLC16 and PLC27) portraying mutations that affected canonical splicing sites (c.1565+1G > A, c.163+2T > G and c.1936+1G > C), which presented negative expression of E-cadherin.

In spite of a high frequency of $A R I D 1 B$ and $A R I D 1 A$ mutations in this series, only one tumour showed absence of expression of ARID1A. Similarly to CDH1, LOH status of the ARID1A region was estimated in the four cases presenting mutations in this gene and we concluded that $\mathrm{LOH}$ could probably occur in only two cases (see Supplementary Table S4). Interestingly, lack of ARID1A expression was confirmed only in the metastatic component of PLC24 (see Figure 4), validating the $\mathrm{LOH}$ status of the ARID1A region in this sample and confirming the pathogenic role of the nonsense p.S634* mutation.

It is important to highlight that mutations in ERBB2 and TP53 did not produced abnormal patterns of IHC features.

\subsection{Molecular Alterations Related to Tumour Progression and Relapse}

For nine patients, we obtained tumour samples from different localizations: the in-situ and the invasive components in four patients; the invasive breast tumour and a lymph node metastasis in three patients; the in-situ and invasive breast components and a lymph node metastasis in one patient and the in-situ primary tumour and the invasive relapse lesion occurring almost five years later in one patient. Comparative results of variants identified in these cases are shown in Supplementary Table S6. Molecular similarities among components highlighted TP53 mutations and amplifications of PIK3CA and CCND1 as progression hallmarks. It is interesting to note that we were able to detect ERBB2 mutations in the in-situ component of two cases of PLC that carried this alteration in the invasive component too. Nine (33\%) out of 27 patients relapsed after a mean follow-up of 44 months; and one (4\%) patient died after 19 months of follow-up. We did not find any pathological feature or molecular alteration associated with tumour relapse. 


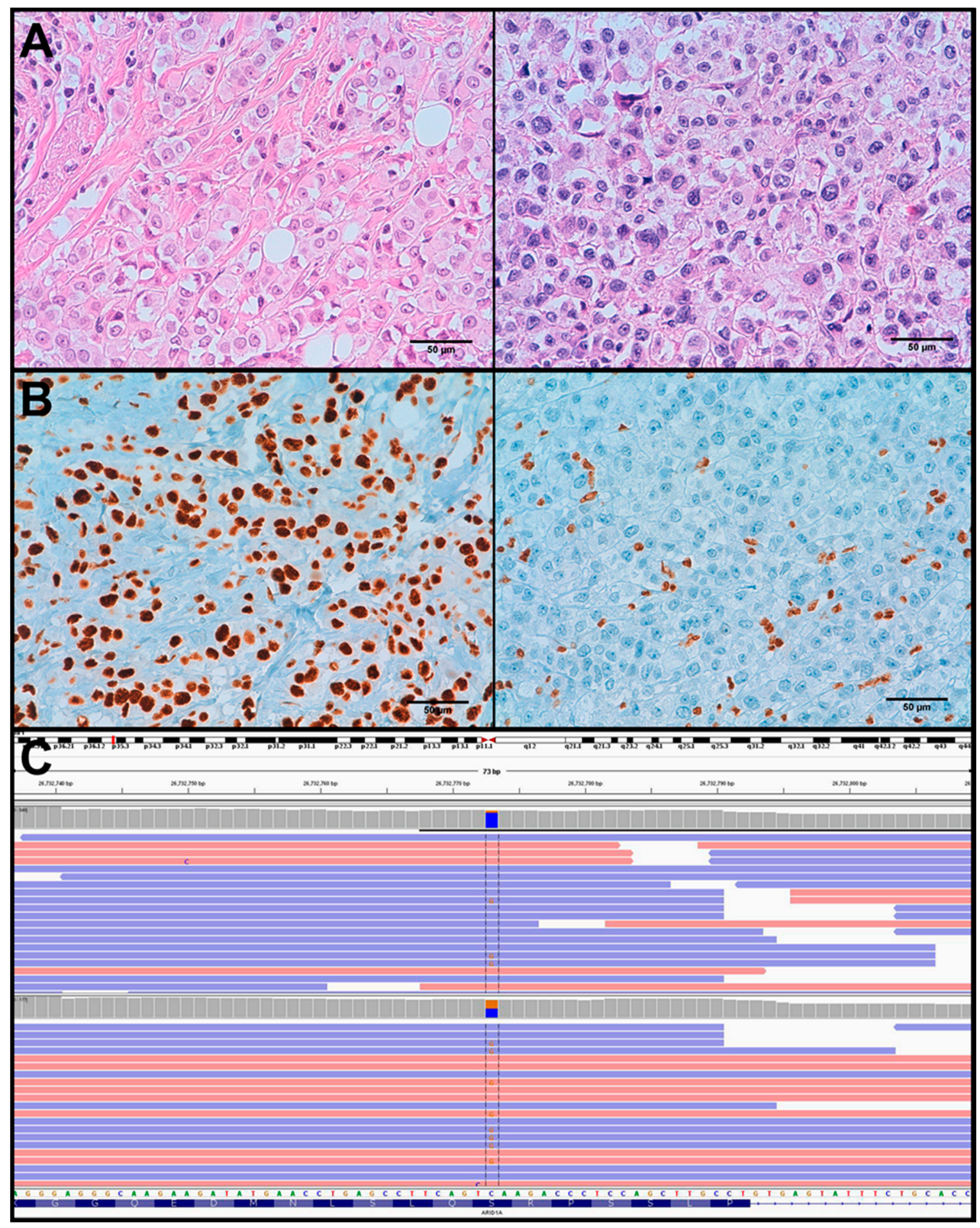

Figure 4. PLC24 sample. (A) H\&E staining of invasive (left) and metastatic (right) components. (B) ARID1A positive staining of invasive component (left) and ARID1A negative staining of metastatic component (right). (C) IGV view of ARID1A S634* mutation frequency (orange) found by NGS sequencing in invasive (top, depth 500) and metastatic (bottom, depth 170) components.

\section{Discussion}

In the current study, the morphological, immunohistochemical and molecular characterisation of a set of invasive lobular carcinomas of the breast with pleomorphic features (at least extensive nuclear grade 3) have all been performed. This study indicates that invasive lobular carcinomas with grade 3 
nuclei are heterogeneous, both at the morphological and molecular levels. In fact, we observed different morphological variants, such as histiocytoid, apocrine and osteoclast-rich carcinomas. All intrinsic subtypes of breast cancer were represented in this series, although most tumours were classified as luminal A with grade 2, probably due to our selection criteria (high nuclear grade and loss or aberrant expression of E-cadherine, independently of the mitotic rate).

Regarding mutational analysis, our study showed some differences with respect to previous NGS studies in ILC. Thus, although CDH1 was the most frequently mutated gene in different series of ILC, the frequency of tumours carrying mutations in $C D H 1$ was about $88 \%$ in our series whereas frequencies varied from $43 \%$ to $65 \%$ in the other studies $[1,8,24]$. These differences are most likely related to case selection rather than to intrinsic biological features of PLC. Thus, all our cases were selected not only according to morphological features but also for the lack or aberrant E-cadherin expression. In this sense, only one of the three previous NGS series analysing ILC did evaluated the immunohistochemical expression of E-cadherin in a limited number of cases, suggesting that possibly not all cases included in previous studies represented bona fide examples of ILC. In addition, even though all PLC in Zhu et al.' series had negative E-cadherin staining, the percentage of cases that carried CDH1 mutations was 59\% [14].

In our series, we did not detect E-cadherin mutation in 3 out of 27 cases with absence of E-cadherin expression. Previous studies suggested that in these cases the loss of E-cadherin expression could be due to a combination of CDH1 LOH and promoter hypermethylation [16]. However, Ciriello et al. has recently questioned this hypothesis, suggesting that promoter hypermethylation is not a feature of ILC [1]. In our series, although we did not studied CDH1 promoter hypermethylation, we estimated the presence of $\mathrm{LOH}$ in $16 \mathrm{q} 22$ in $85 \%$ of the tumours, which is in agreement with previous studies [25].

One interesting finding in our study is the observation of 4 cases $(15 \%)$ with morphological characteristic of PLC, an abnormal pattern of E-cadherin expression and the presence of CDH1 mutations. It has been previously reported that $16 \%$ of ILC retained some E-cadherin expression and that, in these cases, abnormal expression of catenins, such as $\beta$-catenin or p120, was observed [26]. Our study suggested that certain types of $C D H 1$ mutations, especially splice site mutations located in specific domains, are more prone to be related to aberrant E-cadherin expression.

An important finding in our study, with potential therapeutic implications, is the high frequency (26\%) of ERBB2 mutations observed in PLC. Previous NGS studies reported a frequency of ERBB2 mutations in about $5 \%$ of unselected ILC $[1,8,24]$. However, Desmedt et al. reported a correlation between $E R B B 2$ mutations and histological grade, in accordance with our results. In addition, in a study using conventional Sanger sequencing, Lien at al. [10] observed a frequency of $20.8 \%$ of ERBB2 mutations in PLC compared to only a $2 \%$ in classic ILC. Finally, Zhu et al. [14] in their recent NGS study of 16 ILC observed that $E R B B 2$ was mutated in $17 \%$ of the samples. Our results confirmed the important role of $E R B B 2$ gain-of-function mutations in high-grade invasive lobular breast carcinoma, in agreement with previous observations [27]. Of note, ERBB2 activating mutations are considered targetable by anti-ERBB2 drugs [28] and other alternatives could be beneficial for these patients [29]. A comparative study of the frequency of activating mutations in ERBB2 is shown in Table 3. In agreement with previous studies, we did not observe that $E R B B 2$ mutations were associated with a higher risk of relapse [27].

Alterations in the phosphatidylinositol 3-kinase (PI3K) pathway were present in 13 cases (50\%), with mutations in PIK3CA (9 cases, one of them with a combination of gene mutation and amplification), MAP3K1 (5 cases) and AKT1 (2 cases). As expected, mutations in PIK3CA and AKT1 were mutually exclusive [30] but 3 MP3K1 mutations were associated with PIK3CA mutations. Whereas the frequency of PIK3CA mutations in the current series is similar to those reported in other ILC NGS studies, the frequency of $M A P 3 K 1$ mutations seems to be higher (19\% vs. 5-6\%) $[1,8,24]$. This finding is concordant with the study by Zhu et al. reporting that MAP3K1 mutations occurred at a higher frequency in PLC than in ILC [14].

Whereas a unique mutation was found in $A K T 1$ (p.E17K), different known activating mutations were present in PIK3CA (p.E545K, p.E542K and p.H1047R). Moreover, another different case (PLC-8) 
showed the two most frequent mutations in PIK3CA, p.E545K and p.H1047R, a phenomenon that seems to be infrequent. Interestingly, the frequency of the variant alleles in the tumour were $\sim 11 \%$ for p.E545K and $\sim 26 \%$ for p.H1047R, denoting that the former could have been acquired at a later stage in a different chromosome in a specific clone, suggesting clonal diversity.

Table 3. ERBB2 mutations in invasive lobular carcinoma.

\begin{tabular}{ccccccc}
\hline \multirow{2}{*}{ Mene } & Mutation & \multicolumn{2}{c}{ Present Study } & \multicolumn{3}{c}{ Available Studies } \\
\cline { 2 - 6 } & Cases & Frequency & Cases & Frequency & References \\
\hline p.L755S & 4 & $57.1 \%$ & 24 & $33.3 \%$ & {$[1,8,24,27]$} \\
p.L869R & 1 & $14.3 \%$ & 4 & $5.6 \%$ & {$[8]$} \\
p.S310Y & 1 & $14.3 \%$ & 2 & $2.8 \%$ & {$[27]$} \\
p.E717K & 1 & $14.3 \%$ & - & - & - \\
p.V777L & & & 10 & $13.9 \%$ & {$[1,8,14,24]$} \\
p.D769Y & & & 5 & $6.9 \%$ & {$[8,24]$} \\
p.S310F & & & 4 & $5.6 \%$ & {$[8,11,24]$} \\
p.A775_G776insYVMA & & & 3 & $4.2 \%$ & {$[24]$} \\
p.L755_T759del & & & 3 & $4.2 \%$ & {$[10,24]$} \\
p.I767M & & & 2 & $2.8 \%$ & {$[24,27]$} \\
p.R678Q & & & 2 & $2.8 \%$ & {$[1,24]$} \\
c.1647-2A $>$ G & & & 1 & $1.4 \%$ & {$[24]$} \\
c.2923_2923delG & & & 1 & $1.4 \%$ & {$[24]$} \\
p.A771V & & & 1 & $1.4 \%$ & {$[10]$} \\
p.A775V & & & 1 & $1.4 \%$ & {$[10]$} \\
p.E1021K & & & 1 & $1.4 \%$ & {$[8]$} \\
p.L755M & & & 1 & $1.4 \%$ & {$[1]$} \\
p.L755W & & & 1 & $1.4 \%$ & {$[1]$} \\
p.R434Q & & & 1 & $1.4 \%$ & {$[14]$} \\
p.R978C & & 1 & $1.4 \%$ & {$[8]$} \\
p.S305C & & 1 & $1.4 \%$ & {$[1]$} \\
p.T791I & & 1 & $1.4 \%$ & {$[10]$} \\
p.V697L & & 1 & $1.4 \%$ & {$[8]$} \\
p.V842I & & & $1.4 \%$ & {$[24]$} \\
\hline
\end{tabular}

Additionally, several transcriptional regulators were recurrently mutated in the current series, such as ARID1A, ARID1B and KMT2C. Regarding ARID1A and ARID1B, although we observed a high frequency of mutations, the immunohistochemical analysis showed a normal expression pattern in most cases. The discordance between the genomic and the immunohistochemical study can be due to different reasons, such as lack of a second hit (i.e., $\mathrm{LOH}$ ) in most cases, retaining of a non-functional protein that is recognized by the antibodies used, or passenger mutations with no or little impact in gene function. Only one sample with an ARID1A mutation and LOH showed ARID1A loss of expression. Interestingly, loss of ARID1A expression was observed in the lymph node metastasis but not in the primary tumour, suggesting a role of this alteration in tumour progression.

In the TCGA study on breast cancer [31], KTMC2/MLL3 was found to be mutated in the $7 \%$ of cases, with little differences amongst subtypes (5-8\%). These percentages are similar to those reported in other ILC studies $[1,8,24]$. However, the percentage of cases in which we identified mutation in KMT2C was almost $20 \%$, which can contribute to the aggressiveness and dedifferentiation of PLCs due to stemness properties acquired through the loss of KMT2C activity [32]. In the study by Zhu et al. [14], KTMC2 was mutated in $35 \%$ of PLC and this percentage was significantly higher than previously observed in ILC.

In addition to the molecular characterisation of PLCs, we also aimed to check the molecular similarities between different lesions from the same case with the purpose of finding out possible progression hallmarks. As expected, the similarity between in-situ and invasive components of the same case was high, although some differences were shown (see Supplementary Table S6). We observed that mutations in TP53 and amplification of 11q13 region were differential features between invasive 
and in-situ components, highlighting the role of these genes in aggressive tumours as previously stated $[33,34]$.

Germline mutations in BRCA1 and TP53 have been reported to be predominantly associated with invasive ductal carcinomas, while BRCA2 mutations have been related to both ductal and lobular cancers [35]. In addition, a previous study has reported an excess of PLC among BRCA2-mutation carriers [36]. We identified one case out of $27(\sim 4 \%)$ portraying a deleterious germline mutation in $B R C A 2$, with familial history of breast and ovarian cancer. Moreover, we identified a set of infrequent germline mutations with an in-vitro deleteriousness role that are normally excluded from molecular characterisation studies (see Supplementary Table S5). We observed that several variants were not expected to appear in such a small set of cases. Among them, some variants that are considered of "uncertain significance" in familial breast cancer studies testing BRCA1 and BRCA2. Other germline variants were located in ESR1, ERBB2, PIK3CA, KMT2C and ZNF217, genes considered of great importance in the development and outcome of breast cancer $[1,8,31,37-41]$. These infrequent germline variants could be risk factors for the development of PLC, although further studies are necessary to confirm their role in breast cancer development.

\section{Conclusions}

PLC shows a high degree of heterogeneity from a morphological and molecular point of view. In addition to a high percentage of $C D H 1$ mutations, some of which were associated with an aberrant E-cadherin expression pattern, these tumours seem to be characterized by higher frequency of mutations in genes related to aggressive behaviour, such as ERBB2, TP53 and KMT2C. The high frequency of treatable $E R B B 2$ mutations in this and other previous series suggest that $E R B B 2$ mutation testing should be considered in all ILC with nuclear grade 3 .

Supplementary Materials: The following are available online at http://www.mdpi.com/2072-6694/11/1/ 74/s1, Supplementary file 1: Complete mathematical explanations. Table S1: Clinicopathological and immunohistochemical features of the 27 PLC studied, Table S2: List of antibodies used to characterise PLC tumours, Table S3: List of mutations found in the invasive component of the 27 PLCs subjected to NGS analysis, Table S4: Estimation of LOH status based on the calculation of Ti, Table S5: List of infrequent germline variants identified in the 27 PLCs subjected to NGS analysis, Table S6: List of mutations found in the different components of 10 PLCs subjected to NGS analysis.

Author Contributions: Conceptualization, J.M.R.-R. and J.P.; Data curation, S.L.; Formal analysis, J.M.R.-R.; Funding acquisition, P.G. and J.P.; Methodology, J.M.R.-R., T.C.-C., E.C., S.G.-M., E.M.-M. and E.L.-M.; Resources, J.P.; Supervision, E.H., B.P.-M., P.G. and J.P.; Writing—original draft, J.M.R.-R.; Writing—review \& editing, S.L. and J.P.

Funding: This research was funded by Instituto de Salud Carlos III (grant number: PIE15/00050, PI16/00887 and CB16/12/00316), Spanish Association Against Cancer Scientific Foundation (grant number: AIO-AECC 2016) and Fundación la Marató de TV3 (grant number: 2/C2013).

Acknowledgments: We would like to thank Lorezo Melchor for the revision, comments and advices on the final version of this manuscript.

Conflicts of Interest: The authors declare no conflict of interest.

\section{References}

1. Ciriello, G.; Gatza, M.L.; Beck, A.H.; Wilkerson, M.D.; Rhie, S.K.; Pastore, A.; Zhang, H.; McLellan, M.; Yau, C.; Kandoth, C.; et al. Comprehensive Molecular Portraits of Invasive Lobular Breast Cancer. Cell 2015, 163, 506-519. [CrossRef] [PubMed]

2. Lakhani, S.R.; Ellis, I.O.; Schnitt, S.J.; Tan, P.H.; van der Vijver, M.J. WHO Classification of Tumours of the Breast, 4th ed.; World Health Organization: Geneva, Switzerland, 2012.

3. Dixon, J.M.; Anderson, T.J.; Page, D.L.; Lee, D.; Duffy, S.W.; Stewart, H.J. Infiltrating lobular carcinoma of the breast: An evaluation of the incidence and consequence of bilateral disease. Br. J. Surg. 1983, 70, 513-516. [CrossRef] [PubMed]

4. Butler, D.; Rosa, M. Pleomorphic lobular carcinoma of the breast: A morphologically and clinically distinct variant of lobular carcinoma. Arch. Pathol. Lab. Med. 2013, 137, 1688-1692. [CrossRef] [PubMed] 
5. Gamallo, C.; Palacios, J.; Suarez, A.; Pizarro, A.; Navarro, P.; Quintanilla, M.; Cano, A. Correlation of E-cadherin expression with differentiation grade and histological type in breast carcinoma. Am. J. Pathol. 1993, 142, 987-993.

6. Dabbs, D.J.; Schnitt, S.J.; Geyer, F.C.; Weigelt, B.; Baehner, F.L.; Decker, T.; Eusebi, V.; Fox, S.B.; Ichihara, S.; Lakhani, S.R.; et al. Lobular neoplasia of the breast revisited with emphasis on the role of E-cadherin immunohistochemistry. Am. J. Surg. Pathol. 2013, 37, e1-e11. [CrossRef] [PubMed]

7. Sarrio, D.; Moreno-Bueno, G.; Hardisson, D.; Sanchez-Estevez, C.; Guo, M.; Herman, J.G.; Gamallo, C.; Esteller, M.; Palacios, J. Epigenetic and genetic alterations of APC and CDH1 genes in lobular breast cancer: Relationships with abnormal E-cadherin and catenin expression and microsatellite instability. Int. J. Cancer 2003, 106, 208-215. [CrossRef] [PubMed]

8. Desmedt, C.; Zoppoli, G.; Gundem, G.; Pruneri, G.; Larsimont, D.; Fornili, M.; Fumagalli, D.; Brown, D.; Rothe, F.; Vincent, D.; et al. Genomic Characterization of Primary Invasive Lobular Breast Cancer. J. Clin. Oncol. 2016, 34, 1872-1881. [CrossRef]

9. Sarrio, D.; Moreno-Bueno, G.; Sanchez-Estevez, C.; Banon-Rodriguez, I.; Hernandez-Cortes, G.; Hardisson, D.; Palacios, J. Expression of cadherins and catenins correlates with distinct histologic types of ovarian carcinomas. Hum. Pathol. 2006, 37, 1042-1049. [CrossRef]

10. Lien, H.C.; Chen, Y.L.; Juang, Y.L.; Jeng, Y.M. Frequent alterations of HER2 through mutation, amplification, or overexpression in pleomorphic lobular carcinoma of the breast. Breast Cancer Res. Treat. 2015, 150, 447-455. [CrossRef]

11. Reis-Filho, J.S.; Simpson, P.T.; Jones, C.; Steele, D.; Mackay, A.; Iravani, M.; Fenwick, K.; Valgeirsson, H.; Lambros, M.; Ashworth, A.; et al. Pleomorphic lobular carcinoma of the breast: Role of comprehensive molecular pathology in characterization of an entity. J. Pathol. 2005, 207, 1-13. [CrossRef]

12. Simpson, P.T.; Reis-Filho, J.S.; Lambros, M.B.; Jones, C.; Steele, D.; Mackay, A.; Iravani, M.; Fenwick, K.; Dexter, T.; Jones, A.; et al. Molecular profiling pleomorphic lobular carcinomas of the breast: Evidence for a common molecular genetic pathway with classic lobular carcinomas. J. Pathol. 2008, 215, 231-244. [CrossRef] [PubMed]

13. Dieci, M.V.; Smutna, V.; Scott, V.; Yin, G.; Xu, R.; Vielh, P.; Mathieu, M.C.; Vicier, C.; Laporte, M.; Drusch, F.; et al. Whole exome sequencing of rare aggressive breast cancer histologies. Breast Cancer Res. Treat. 2016, 156, 21-32. [CrossRef] [PubMed]

14. Zhu, S.; Ward, B.M.; Yu, J.; Matthew-Onabanjo, A.N.; Janusis, J.; Hsieh, C.C.; Tomaszewicz, K.; Hutchinson, L.; Zhu, L.J.; Kandil, D.; et al. IRS2 mutations linked to invasion in pleomorphic invasive lobular carcinoma. JCI Insight 2018, 3. [CrossRef] [PubMed]

15. Wolff, A.C.; Hammond, M.E.H.; Allison, K.H.; Harvey, B.E.; Mangu, P.B.; Bartlett, J.M.S.; Bilous, M.; Ellis, I.O.; Fitzgibbons, P.; Hanna, W.; et al. Human Epidermal Growth Factor Receptor 2 Testing in Breast Cancer: American Society of Clinical Oncology/College of American Pathologists Clinical Practice Guideline Focused Update. J. Clin. Oncol. 2018, 36, 2105-2122. [CrossRef] [PubMed]

16. Sarrio, D.; Perez-Mies, B.; Hardisson, D.; Moreno-Bueno, G.; Suarez, A.; Cano, A.; Martin-Perez, J.; Gamallo, C.; Palacios, J. Cytoplasmic localization of p120ctn and E-cadherin loss characterize lobular breast carcinoma from preinvasive to metastatic lesions. Oncogene 2004, 23, 3272-3283. [CrossRef] [PubMed]

17. Curigliano, G.; Burstein, H.J.; Winer, E.P.; Gnant, M.; Dubsky, P.; Loibl, S.; Colleoni, M.; Regan, M.M.; Piccart-Gebhart, M.; Senn, H.J.; et al. De-escalating and escalating treatments for early-stage breast cancer: The St. Gallen International Expert Consensus Conference on the Primary Therapy of Early Breast Cancer 2017. Ann. Oncol. 2017, 28, 1700-1712. [CrossRef] [PubMed]

18. Maisonneuve, P.; Disalvatore, D.; Rotmensz, N.; Curigliano, G.; Colleoni, M.; Dellapasqua, S.; Pruneri, G.; Mastropasqua, M.G.; Luini, A.; Bassi, F.; et al. Proposed new clinicopathological surrogate definitions of luminal A and luminal B (HER2-negative) intrinsic breast cancer subtypes. Breast Cancer Res. 2014, 16, R65. [CrossRef] [PubMed]

19. Rosa-Rosa, J.M.; Caniego-Casas, T.; Leskela, S.; Munoz, G.; Del Castillo, F.; Garrido, P.; Palacios, J. Modified SureSelect(QXT) Target Enrichment Protocol for Illumina Multiplexed Sequencing of FFPE Samples. Biol. Proced. Online 2018, 20, 19. [CrossRef]

20. Shigemizu, D.; Momozawa, Y.; Abe, T.; Morizono, T.; Boroevich, K.A.; Takata, S.; Ashikawa, K.; Kubo, M.; Tsunoda, T. Performance comparison of four commercial human whole-exome capture platforms. Sci. Rep. 2015, 5, 12742. [CrossRef] 
21. Koboldt, D.C.; Zhang, Q.; Larson, D.E.; Shen, D.; McLellan, M.D.; Lin, L.; Miller, C.A.; Mardis, E.R.; Ding, L.; Wilson, R.K. VarScan 2: Somatic mutation and copy number alteration discovery in cancer by exome sequencing. Genome Res. 2012, 22, 568-576. [CrossRef]

22. Li, J.; Lupat, R.; Amarasinghe, K.C.; Thompson, E.R.; Doyle, M.A.; Ryland, G.L.; Tothill, R.W.; Halgamuge, S.K.; Campbell, I.G.; Gorringe, K.L. CONTRA: Copy number analysis for targeted resequencing. Bioinformatics 2012, 28, 1307-1313. [CrossRef]

23. Pena-Jaimes, L.; Gonzalez-Garcia, I.; Reguero-Callejas, M.E.; Pinilla-Pagnon, I.; Perez-Mies, B.; Albarran-Artahona, V.; Martinez-Janez, N.; Rosa-Rosa, J.M.; Palacios, J. Pleomorphic lobular carcinoma of the breast with osteoclast-like giant cells: A case report and review of the literature. Diagn. Pathol. 2018, 13, 62. [CrossRef] [PubMed]

24. Michaut, M.; Chin, S.F.; Majewski, I.; Severson, T.M.; Bismeijer, T.; de Koning, L.; Peeters, J.K.; Schouten, P.C.; Rueda, O.M.; Bosma, A.J.; et al. Integration of genomic, transcriptomic and proteomic data identifies two biologically distinct subtypes of invasive lobular breast cancer. Sci. Rep. 2016, 6, 18517. [CrossRef]

25. Palacios, J.; Sarrio, D.; Garcia-Macias, M.C.; Bryant, B.; Sobel, M.E.; Merino, M.J. Frequent E-cadherin gene inactivation by loss of heterozygosity in pleomorphic lobular carcinoma of the breast. Mod. Pathol. 2003, 16, 674-678. [CrossRef] [PubMed]

26. Rakha, E.A.; Patel, A.; Powe, D.G.; Benhasouna, A.; Green, A.R.; Lambros, M.B.; Reis-Filho, J.S.; Ellis, I.O. Clinical and biological significance of E-cadherin protein expression in invasive lobular carcinoma of the breast. Am. J. Surg. Pathol. 2010, 34, 1472-1479. [CrossRef]

27. Deniziaut, G.; Tille, J.C.; Bidard, F.C.; Vacher, S.; Schnitzler, A.; Chemlali, W.; Tremoulet, L.; Fuhrmann, L.; Cottu, P.; Rouzier, R.; et al. ERBB2 mutations associated with solid variant of high-grade invasive lobular breast carcinomas. Oncotarget 2016, 7, 73337-73346. [CrossRef] [PubMed]

28. Bidard, F.C.; Ng, C.K.; Cottu, P.; Piscuoglio, S.; Escalup, L.; Sakr, R.A.; Reyal, F.; Mariani, P.; Lim, R.; Wang, L.; et al. Response to dual HER2 blockade in a patient with HER3-mutant metastatic breast cancer. Ann. Oncol. 2015, 26, 1704-1709. [CrossRef]

29. Li, G.; Wang, X.; Hibshoosh, H.; Jin, C.; Halmos, B. Modulation of ErbB2 blockade in ErbB2-positive cancers: The role of ErbB2 Mutations and PHLDA1. PLoS ONE 2014, 9, e106349. [CrossRef]

30. Bleeker, F.E.; Felicioni, L.; Buttitta, F.; Lamba, S.; Cardone, L.; Rodolfo, M.; Scarpa, A.; Leenstra, S.; Frattini, M.; Barbareschi, M.; et al. AKT1(E17K) in human solid tumours. Oncogene 2008, 27, 5648-5650. [CrossRef]

31. Cancer Genome Atlas Research Network. Comprehensive molecular characterization of clear cell renal cell carcinoma. Nature 2013, 499, 43-49. [CrossRef]

32. Zhang, Z.; Christin, J.R.; Wang, C.; Ge, K.; Oktay, M.H.; Guo, W. Mammary-Stem-Cell-Based Somatic Mouse Models Reveal Breast Cancer Drivers Causing Cell Fate Dysregulation. Cell Rep. 2016, 16, 3146-3156. [CrossRef] [PubMed]

33. Rosa-Rosa, J.M.; Leskela, S.; Cristobal-Lana, E.; Santon, A.; Lopez-Garcia, M.A.; Munoz, G.; Perez-Mies, B.; Biscuola, M.; Prat, J.; Esther, O.E.; et al. Molecular genetic heterogeneity in undifferentiated endometrial carcinomas. Mod. Pathol. 2016, 29, 1594. [CrossRef] [PubMed]

34. Ahlin, C.; Lundgren, C.; Embretsen-Varro, E.; Jirstrom, K.; Blomqvist, C.; Fjallskog, M. High expression of cyclin D1 is associated to high proliferation rate and increased risk of mortality in women with ER-positive but not in ER-negative breast cancers. Breast Cancer Res. Treat. 2017, 164, 667-678. [CrossRef]

35. Dossus, L.; Benusiglio, P.R. Lobular breast cancer: Incidence and genetic and non-genetic risk factors. Breast Cancer Res. 2015, 17, 37. [CrossRef]

36. Armes, J.E.; Egan, A.J.; Southey, M.C.; Dite, G.S.; McCredie, M.R.; Giles, G.G.; Hopper, J.L.; Venter, D.J. The histologic phenotypes of breast carcinoma occurring before age 40 years in women with and without BRCA1 or BRCA2 germline mutations: A population-based study. Cancer 1998, 83, 2335-2345. [CrossRef]

37. Hernandez, L.; Wilkerson, P.M.; Lambros, M.B.; Campion-Flora, A.; Rodrigues, D.N.; Gauthier, A.; Cabral, C.; Pawar, V.; Mackay, A.; A'Hern, R.; et al. Genomic and mutational profiling of ductal carcinomas in situ and matched adjacent invasive breast cancers reveals intra-tumour genetic heterogeneity and clonal selection. J. Pathol. 2012, 227, 42-52. [CrossRef] [PubMed]

38. Schonleben, F.; Qiu, W.; Ciau, N.T.; Ho, D.J.; Li, X.; Allendorf, J.D.; Remotti, H.E.; Su, G.H. PIK3CA mutations in intraductal papillary mucinous neoplasm/carcinoma of the pancreas. Clin. Cancer Res. 2006, 12, 3851-3855. [CrossRef] 
39. Bellanger, A.; Donini, C.F.; Vendrell, J.A.; Lavaud, J.; Machuca-Gayet, I.; Ruel, M.; Vollaire, J.; Grisard, E.; Gyorffy, B.; Bieche, I.; et al. The critical role of the ZNF217 oncogene in promoting breast cancer metastasis to the bone. J. Pathol. 2017, 242, 73-89. [CrossRef]

40. Maatta, K.; Rantapero, T.; Lindstrom, A.; Nykter, M.; Kankuri-Tammilehto, M.; Laasanen, S.L.; Schleutker, J. Whole-exome sequencing of Finnish hereditary breast cancer families. Eur. J. Hum. Genet. 2016, 25, 85-93. [CrossRef]

41. Xie, Y.; Li, G.; Chen, M.; Guo, X.; Tang, L.; Luo, X.; Wang, S.; Yi, W.; Dai, L.; Wang, J. Mutation screening of 10 cancer susceptibility genes in unselected breast cancer patients. Clin. Genet. 2017. [CrossRef]

(C) 2019 by the authors. Licensee MDPI, Basel, Switzerland. This article is an open access article distributed under the terms and conditions of the Creative Commons Attribution (CC BY) license (http://creativecommons.org/licenses/by/4.0/). 